\title{
Osteoblastoma con presentación atípica en cuboides
}

\author{
Osteoblastoma with atypical presentation in cuboid bone
}

\author{
Moreno-Hoyos LF,* Strassburger-Weidmann J, ${ }^{\ddagger}$ Castillo-Anaya V, ${ }^{\S}$ Galindo-Agustín LO^ \\ Hospital Ángeles Mocel.
}

RESUMEN. Introducción: El osteoblastoma es un tumor osteoblástico benigno, agresivo y poco frecuente. Su localización más frecuente es en elementos posteriores de la columna vertebral y el sacro. La presentación en cuboides es excepcional. Caso clínico: Masculino de 50 años que inició padecimiento en 2005, con dolor y aumento de volumen en región dorsolateral del mediopié. En las radiografías se observa injerto óseo y una lesión en cuboides, radiotransparente, heterogénea, multilobulada, con bordes irregulares que sobrepasa la cortical lateral. Se realizó angiotomografía donde se observó la lesión hipervascularizada; en la gammagrafía se observó captación del tecnecio 99 y en la resonancia magnética se reporta una lesión con cambios postquirúrgicos, quística, multilobulada. Se tomó biopsia transquirúrgica con abundante tejido fibroconectivo, osteoblastos, nidos de tejido osteoide e hipervascularidad del estroma, aumento de celularidad sin atipias y escasas células gigantes multinucleadas. Se clasificó Enneking 2. Se inició tratamiento con resección y curetaje del tumor, crioterapia y colocación de injerto tricortical en el defecto óseo. Mostró buena evolución postquirúrgica. A los tres años del procedimiento quirúrgico se encuentra sin datos de actividad tumoral y asintomático. A pesar de que el osteoblastoma no se presenta de forma habitual en cuboides, debe tomarse en cuenta como diagnóstico diferencial. Conclusión: Los tumores óseos, a pesar de tener localizaciones habituales, pueden presentarse en zonas poco frecuentes y por lo tanto, el estudio completo clínico radiográfico e histopatológico en cada paciente es fundamental.

Palabras clave: Osteoblastoma, cuboides, tumor óseo, tratamiento, diagnóstico.
ABSTRACT. Introduction: Osteoblastoma is a benign, aggressive and rare osteoblastic tumor. Its most common location is in later elements of the spine and sacrum. The cuboid presentation is exceptional. Case report: A 50-yearold male who began her condition in 2005, with pain and increased volume in the dorsolateral region of the middle foot. X-rays show bone grafting and a cuboid lesion, radiotransparent, heterogeneous, multilobed, with irregular edges that exceeds the lateral cortical. Angiotomography was performed where hypervascularized injury was observed; bone scan showed uptake of technetium 99, and MRI reported an injury with post-surgical, cystic and multilobed changes. Trans surgical biopsy was taken, increased atypiafree cellularity and few multinucleated giant cells were reported and Enneking 2 qualified. Treatment was initiated with resection of the tumor, cryotherapy, and placement of tricortical graft in the bone defect. He exhibited good post-surgical evolution. At three years of the surgical procedure, he`s without tumor and asymptomatic. Although osteoblastoma does not usually occur in cuboid, it should be taken into account as a differential diagnosis. Conclusion: Bone tumors, despite having common locations, can occur in rare areas and therefore the complete radiographic and histopathological clinical study in each patient is critical.

Keywords: Osteoblastoma, cuboid, bone tumor, treatment, diagnosis.

\footnotetext{
* Médico adscrito de Ortopedia y Traumatología del Hospital Ángeles Mocel. Especialista en Tumores Óseos.

* Médico Especialista en Ortopedia y Traumatología, en Curso de Alta Especialidad en Cirugía Articular y Artroscopía en el Hospital Regional de Tlalnepantla del ISSEMyM.

${ }^{\S}$ Médico residente de Ortopedia y Traumatología del Hospital Ángeles Mocel, avalada por la Facultad Mexicana de Medicina de la Universidad La Salle.

^ Médico adscrito de Ortopedia y Traumatología del Hospital Ángeles Mocel.
}

Correspondencia:

E-mail: drmorenohoyos@yahoo.com 


\section{Introducción}

El osteoblastoma es un tumor osteoblástico benigno, agresivo y poco frecuente. ${ }^{1,2,3,4,5}$ Comprende $3 \%$ de todos los tumores óseos benignos y alrededor de $1 \%$ de todos los tumores óseos primarios. Tiene mayor incidencia entre 10 y 25 años de edad y una proporción 2:1 entre masculino y femenino. ${ }^{1,2,3,4,5}$ Desde el punto de vista clínico se caracteriza por un dolor latente, continuo, lentamente progresivo, el cual no cede con ácido acetilsalicílico y en algunas ocasiones puede producir síntomas neurológicos cuando se localiza en los elementos posteriores de la columna vertebral. . $^{1,2,3,4,5,6}$ Puede producir tumefacción, atrofia muscular y alteraciones en la marcha. ${ }^{1,2,3,4,5,6}$ Por medio de estudios de imagen suele presentarse como una lesión radiotransparente de 2 a $10 \mathrm{~cm}$ de tamaño con heterogeneidad de densidad en su interior; son tumores expansivos con extensión a partes blandas circundantes $\mathrm{y}$ un halo de hueso reactivo alrededor del tumor. ${ }^{2,3,6}$ Las radiografías simples pueden dar de 43 a 66\% de certeza en el diagnóstico y de 15 a 33\% pueden pasar como diagnóstico diferencial de lesiones malignas como osteosarcoma. ${ }^{2,7}$ La tomografía computarizada es el método de imagen de elección, ya que puede otorgar la información más específica en cuanto a localización, tamaño, extensión y naturaleza del tumor. ${ }^{2,3,5,6}$ La tomografía muestra típicamente áreas de mineralización central, remodelación ósea, esclerosis reactiva periférica. ${ }^{2,3,5,6}$ La resonancia magnética puede ser útil en caso de duda en el diagnóstico. ${ }^{2,3,5,6}$ En el estudio histopatológico se observan ramificaciones irregulares de osteoide separadas por un estroma fibrovascular laxo; el osteoide rodeado de osteoblastos prominentes grandes y epiteloides, entre $10 \mathrm{y}$ $40 \%$ de estos tumores están relacionados con la formación de un quiste óseo aneurismático secundario. ${ }^{1,2,6,8} \mathrm{El}$ tratamiento que se realiza es el curetaje intralesional o la resección en bloque, dependiendo las características de la lesión. ${ }^{2,9,10,11,12,13}$ Su localización más frecuente es en los

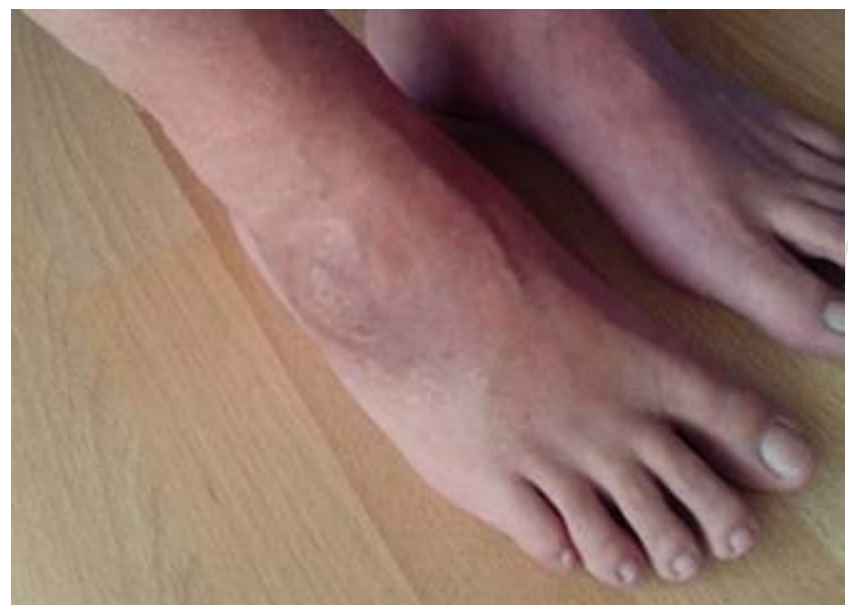

Figura 1: Presentación clínica.

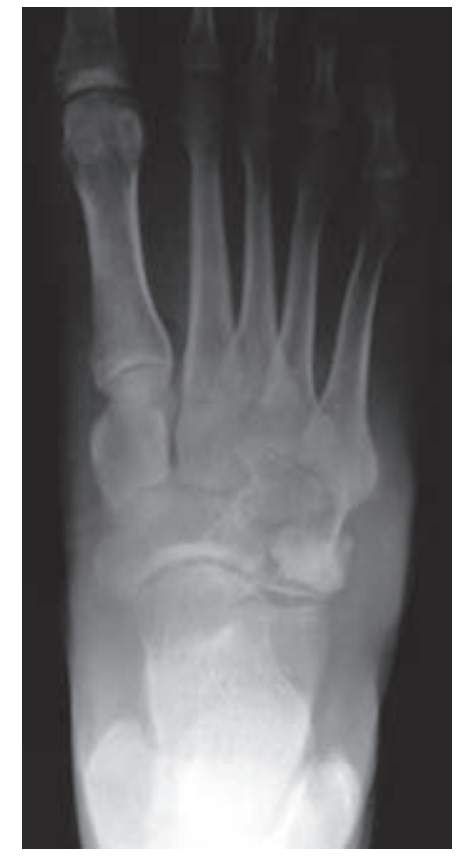

Figura 2:

Radiografía dorsoplantar de pie.

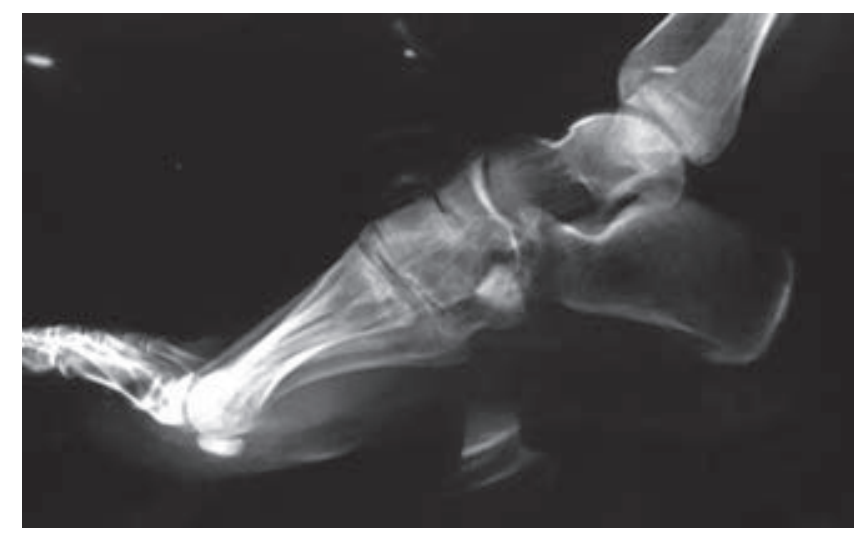

Figura 3: Radiografía lateral de pie.

elementos posteriores de la columna vertebral y el sacro y en menor proporción en diáfisis y metáfisis de huesos largos y en mandíbula; ; ${ }^{1,2,4,8,9,10}$ no obstante, la presentación en cuboides se describe en algunos artículos como una localización excepcional, pero con algunos casos reportados. ${ }^{11,12,14,15}$ Se presenta el caso de un osteoblastoma en localización en cuboides manejado con resección y curetaje del tumor, crioterapia y colocación de injerto tricortical en el defecto y con tres años de seguimiento tras concluido el tratamiento.

\section{Caso clínico}

Se trata de paciente del género masculino de 50 años de edad con antecedente de tabaquismo a expensas de 10 cigarrillos al día por 34 años, resto interrogado y negado. El cuadro clínico inició en el año 2005, al ir corriendo, sin mecanismo de lesión traumático aparente, con dolor 
súbito en dorso de mediopié derecho, de predominio lateral, continuo, opresivo, progresivo, con intensidad ocho de 10, irradiado a región plantar ipsilateral, excacerbado con la deambulación y atenuado parcialmente con nimesulida. Acude con médico, el cual realiza estudios de gabinete y en 2006 se lleva a cabo una cirugía de curetaje y colocación de injerto óseo, persistiendo las molestias, se efectuó un segundo procedimiento para revisar la lesión y una tenoplastía de los extensores de los dedos, ya que se observó la ruptura de los mismos. Posterior al segundo procedimiento quirúrgico permanecieron las molestias y en Diciembre de 2014 acude para una nueva evaluación y se inicia protocolo de estudio del caso. A la exploración del pie derecho se observa aumento de volumen localizado en región lateral de mediopié con bordes bien delimitados y regulares, doloroso a la palpación, de un tamaño de $4 \times 3 \mathrm{~cm}$, dos cicatrices de procedimientos anteriores. (Figura 1).

En las radiografías dorsoplantar y lateral de pie derecho se observa injerto óseo en el centro de una lesión en cuboides, radiotransparente, heterogénea, multilobulada, con bordes irregulares que sobrepasan la cortical lateral, multilobulados (Figuras 2 y 3 ).

Como estudios complementarios se encontró en la angiotomografía evidencia de la lesión hipervascularizada en casi todo el cuboides; en la gammagrafía con Tecnecio 99 se observa una captación del radiomarcador y la resonancia magnética reportó cambios postquirúrgicos en cuboides y una lesión ósea de aspecto quístico multilobulado.

Se realizó la biopsia transquirúrgica, la cual reveló abundante tejido fibroconectivo con osteoblastos y nidos de hueso trabecular con matriz ósea y tejido osteoide e hipervascularidad del estroma, aumento de la celularidad sin atipias y escasas células gigantes multinucleadas (Figura 4); se clasificó como Enneking 2. Se inició tratamiento con resección y curetaje del tumor, crioterapia y colocación de injerto tricortical en el defecto en cuboides (Figura 5).

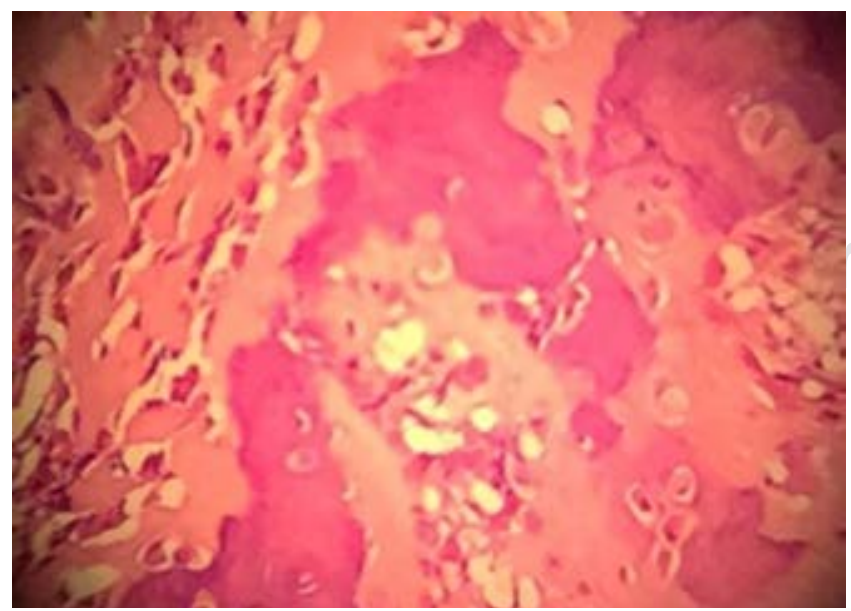

Figura 4: Resultado histopatológico microscópico.
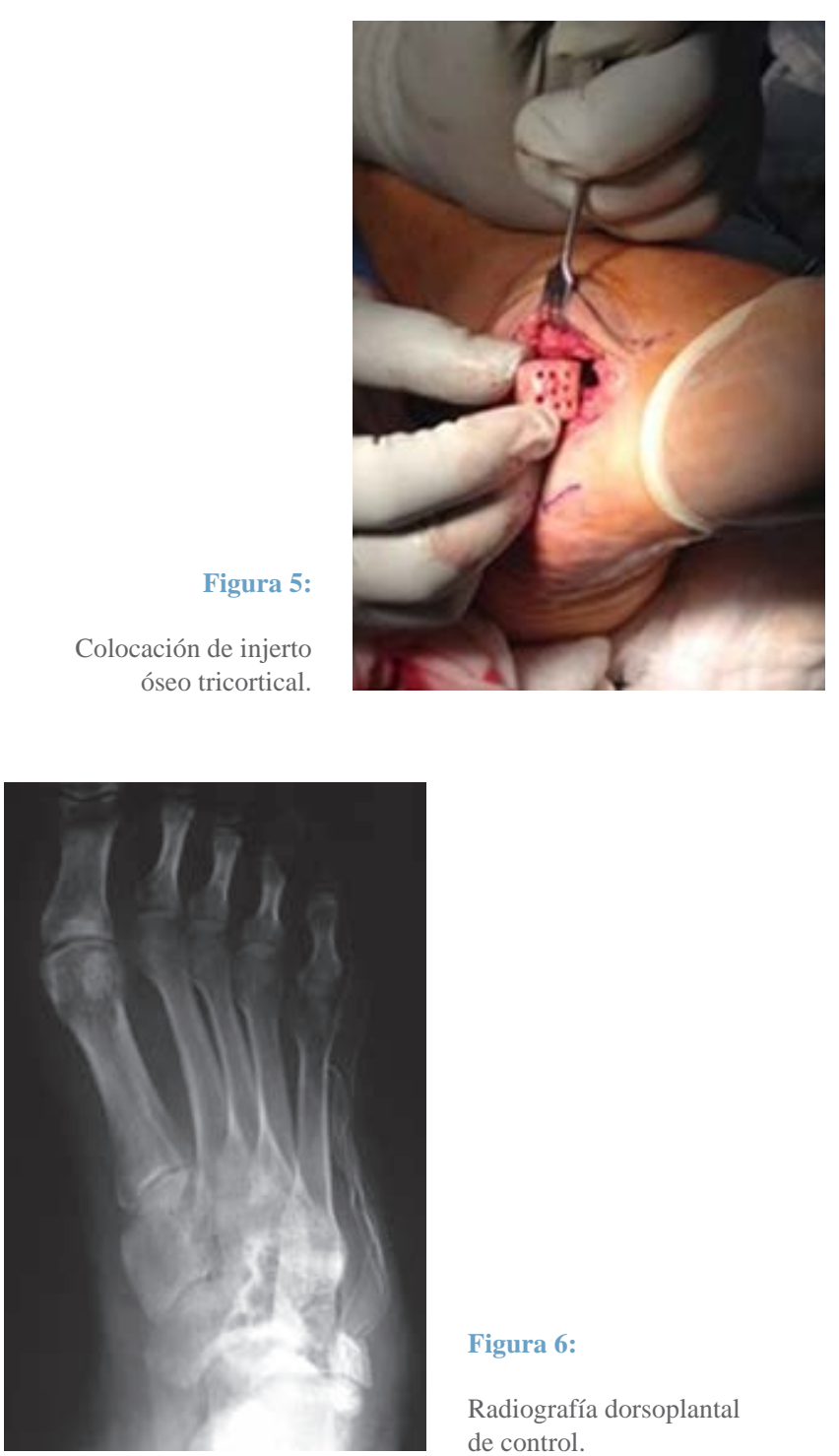

Figura 6:

Radiografía dorsoplantal de control.

Mostró buena evolución postquirúrgica clínica y radiográfica (Figuras 6 y 7). A los tres años del procedimiento quirúrgico se encuentra sin datos de actividad tumoral local o a distancia, tolerando la deambulación sin auxiliares de la marcha y asintomático.

\section{Discusión}

Pese a que el osteoblastoma no se presenta de forma habitual en cuboides, ${ }^{11,12,14,15}$ debe siempre tomarse en cuenta como un diagnóstico diferencial, ${ }^{7}$ ya que a pesar de ser una localización excepcional, se han encontrado algunos casos en la literatura ${ }^{11,12,14,15}$ del mismo modo en que se presentó en este caso.

\section{Conclusión}

Esta presentación de osteoblastoma en cuboides nos demuestra que los tumores óseos, a pesar de tener localizacio- 


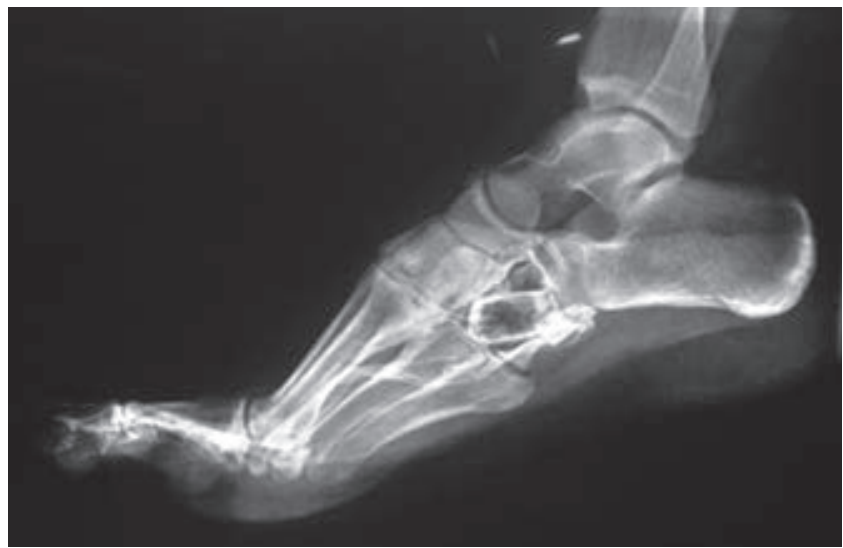

Figura 7: Radiografía lateral de control.

nes y presentaciones habituales, pueden aparecer en formas poco frecuentes y por lo tanto, el estudio completo clínico, radiográfico e histopatológico en cada paciente es fundamental.

\section{Referencias}

1. Lichtenstein L. Benign osteoblastoma; a category of osteoid-and bone-forming tumors other than classical osteoid osteoma, which may be mistaken for giant-cell tumor or osteogenic sarcoma. Cancer. 1956; 9: 1044-52.

2. Atesok KI, Alman BA, Schemitsch EH, Peyser A, Mankin H. Osteoid osteoma and obsteoblastoma. J Am Acad Orthop Surg. 2011; 19(11): 678-89.
3. Greenspan A. Benign bone-forming lesions: osteoma, osteoid osteoma, and osteoblastoma. Clinical, imaging, pathologic, and differential considerations. Skeletal Radiol. 1993; 22(7): 485-500.

4. Oliveira CR, Mendonca BB, Camargo OP, Pinto EM, Nascimento SA, Latorre Mdo R, et al. Classical osteoblastoma, atypical osteoblastoma, and osteosarcoma: a comparative study based on clinical, histological, and biological parameters. Clinics (Sao Paulo). 2007; 62(2): 167-74.

5. Rosensweig J, Pintar K, Mikail M, Mayman A. Benign Osteoblastoma (giant osteoid osteoma): report of an unusual rib tumour and review of the literature. Can Med Assoc J. 1963; 89: 1189-92.

6. Lucas DR. Osteoblastoma. Arch Pathol Lab Med. 2010; 134(10): 1460-6.

7. Kumar NL, Rosenberg AE, Raskin KA. Osteoblastoma-like osteosarcoma of the cuboid: a case report. J Orthop Surg Res. 2010; 5(1): 52.

8. Galgano MA, Goulart CR, Iwnofu H, Chin LS, Lavelle W, Mendel E. Osteoblastomas of the spine: a comprehensive review. Neurosurg Focus. 2016; 41(2): E4.

9. Berry M, Mankin H, Gebhardt M, Rosenberg A, Hornicek F. Osteoblastoma: a 30-year study of 99 cases. J Surg Oncol. 2008; 98(3): 179-83.

10. Elder BD, Goodwin CR, Kosztowski TA, Lo SF, Bydon A, Wolinsky JP, et al. Surgical management of osteoblastoma of the spine: case series and review of the literature. Turk Neurosurg. 2016; 26(4): 601-7.

11. McLeod RA, Dahlin DC, Beabout JW. The spectrum of osteoblastoma. AJR Am J Roentgenol. 1976; 126(2): 321-5.

12. Ouabid A, Etiun P, Goldberg F, Jennart H, De Roover R, Chojnowski J, et al. L'ostéoblastome du cuboide: une localisation exceptionnelle. Med Chir Pied. 2009; 25: 66-8.

13. Weber MA, Sprengel SD, Omlor GW, Lehner B, Wiedenhofer B, Kauczor HU, et al. Clinical long-term outcome, technical success, and cost analysis of radiofrequency ablation for the treatment of osteoblastomas and spinal osteoid osteomas in comparison to open surgical resection. Skeletal Radiol. 2015; 44: 981-93.

14. Schajowicz F, Lemos C. Osteoid osteoma and osteoblastoma, Closely related entities of osteoblastic derivation. Acta Orthop Scandinav. 1970; 41: 272-91.

15. Temple HT, Mizel MS, Murphey MD, Sweet DE. Osteoblastoma of the foot and ankle. Foot Ankle Int. 1998; 19(10): 698-704. 